\title{
ANALOGIE ENTRE MARÉES ET COURANTS ALTERNATIFS
}

\author{
Dr Johan VA.N VEEN \\ Rijkswalerstaat, Hullande
}

NOTATIONS (Voir figures a ef $b$ )

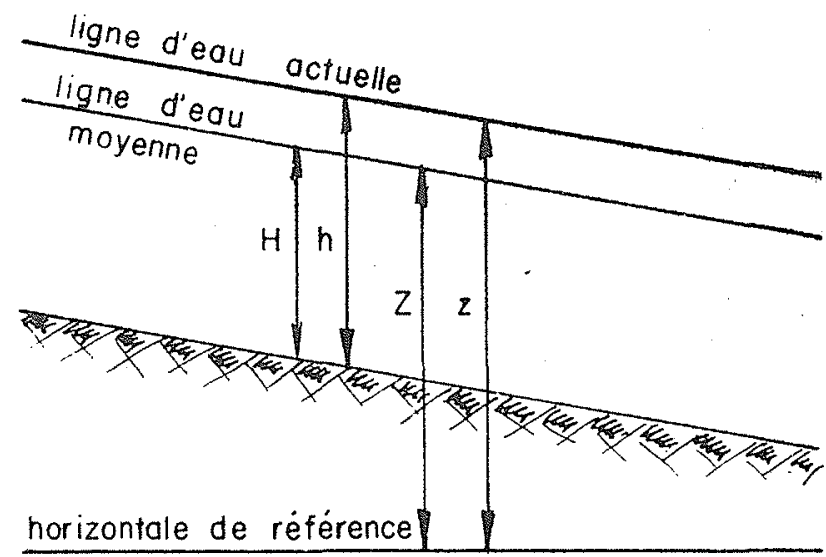

longhueul.

hautenr.

profondent moyenne d'un tronçon de rivière it marée.

profondewr moyenne.

hauteur d'eau au-dessus d'une horizontale.

lageur du bassin de maree.

largeur du courant dans un chenal.

temps.

vitesse du commat.

débit moyen par seconde.

accélemation de la pesanteur.

coefficient de travail.

l'ésistance.

constante.

aire d'un profil.

proportionnel a.

fig. a

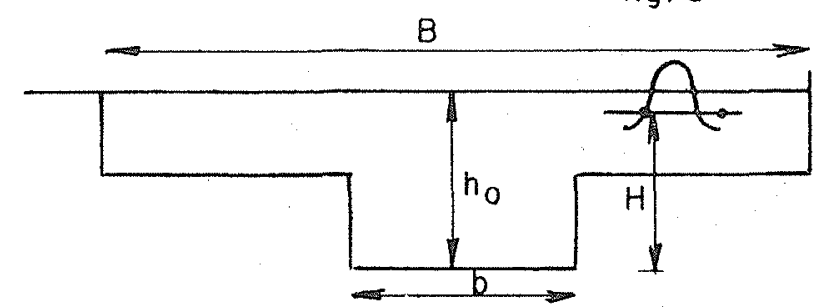

fig. $b$

amplitude verticale de la maree (entre Pal of BM) ou marnage.

surface mouillée d'un profil de rivièro.

périmètre mouillé.

rayon hydraulique.

surface d'un bassin ou d'un trongon de rivière.

evasement, par lim, d'une rivière à maré.

débit instantane d'une rivière.

débit d'une piviere pendant un eycle de maree $(44.700 \mathrm{sec}$ )

volume total ecoule pendant le flot.

volume tolal écoulé pendant le jusant.

$-e+f$ volume total echangé au cours d'un eycle de mare (fitol et jusant:

- abstraction faite du sens d'écoulement.

distance séparant deux sections $A$ et $B$.

pente, puis gradient moteur $\mathrm{H} / 1$.

temps de propagation de $A$ en $B$.

célérité ou vitesse de propagation entre $A$ of $\mathrm{B}$

facteur d'activile.

aire motrice par evele entre $A \& 1 \quad B$ 


\section{CHAPITRE I}

On admet, généralement, la difficulté du calcu! exact des marées dans les nouveaux canaux. Ail paragraphe I nous montrerons qu'il en est bien ainsi. Au paragraphe II, nous exposerons une analogie simple gràce à laquelle on peut se livre: à des calculs élémentaires. Cela conduira, a! paragraphe III, à des solutions électriques expérimentales. La démonstration des formules est donnée dans la bibliographie du paragraphe IV.

Si nous laissons de còté quelques précoces tentatives en vue d'une résolution par la voie mathématique du problème des marées dans un réseau de canaux, c'est te Prof. H.-A. Lonentz, de Leiden, auquel on doit la découverte des électrons, qui donna le premier deux relations scientifiques et d'usage pratique, à savoir :

$\frac{1}{g / \mathrm{H}} \frac{\delta s}{\delta t}=-\frac{\delta h}{\delta x}-\frac{K s}{f H}$ (équation dynamique) $\frac{\delta s}{\delta x}=-\mathrm{I} \frac{\delta h}{\delta t}$ (équation de continuité)

Ces relations, qu'il faut utiliser simultanément, découlent de l'idée que deux séries d'ondes sinusoïdales se propagent, au mème instant, dans deux directions opposées.

Nous n'essayerons pas de justifier ces deux. relations : cela nous conduirait trop loin. On trouvera, d'ailleurs, des explications détaillées dans le livre du Comité du Zuiderzée, Comité dont Loreñaz était Président. Il fallut huit ans pour résoudre le problème des marées du Zuiderzée à l'aide de ces relations (fig. 1).

Les simplifications qu'il fallait admettre ètaient la forme sinusoïdale de l'onde de marée et l'existence d'une relation linéaire entre ia vitesse du courant et le frottement, à savoir : $R_{1} \infty \mathrm{KV}$, formule dans laquelle $\mathrm{K}$ est une certaine constante.

Cependant, la relation exacte serait :

$$
\mathrm{R} c 0 \frac{\mathrm{V}^{2}}{\mathrm{C}^{2}}
$$

En égalant les travaux de $R$, et de $R$ au cours d'un cycle de marée, Lorentz put opérer avec une exactitude suffisante, tandis qu'il maintenait ses équations linéaires, donc solubles.

La pratique confirma ses idées. Après la fermeture du Zuiderzée, la marée devait s'accroìtre selon les calculs, et les courants dans les goulets des îles Frisonnes devinrent d'environ $20 \%$ supérieurs aux prévisions des calculs. Un raisonnement superficiel aurait pu aboutir à la conclusion erronée d'un affaiblissement de ces courants après amputation d'une si grande superficie ou du bassin livré à la marée.
Les équations de LonswTz ne pourraient pas ĉtre utilisées pour les problèmes d'estuaires de fleures à marées, à cause de la pente créée dan', ces estuaires par le débit du fleuve, et par-dessus laquelle l'onde de marée se propage. C'est pourquoi J.-P. Mazure, Ingénieur des Travaux du Zuiderzée, généralisa ces équations de la manière suivante :

$$
\begin{gathered}
\frac{\delta z}{\delta x}=-\frac{1}{g l} \frac{\delta s}{\delta l}+\frac{s}{g l^{2}}\left(\frac{\delta l}{\delta l}+b \frac{\delta h}{\delta l}\right) \\
+\frac{s^{2}}{g / 3} \frac{\delta l}{\delta x}-\frac{s}{C} \frac{s}{C R} \frac{\delta s}{\delta x}=-\mathrm{B} \frac{\delta h}{\delta l}
\end{gathered}
$$

Comme précédemment, ces formules ne sont valables que pour des marées purement sinusoïdales et supposent également la relation linéaire.

Pour obtenir une exactitude encore plus grande, le bureau des recherches du Rijkswaterslaat a mis au point une troisième paire de relations, à vrai dire les premières exactes. An moyen d'une série, le mathématicien JJ. DronkERs put introduire la relation quadratique et les marées naturelles non sinusoïdales. Ces formules dites « exactes " sont :

$$
\begin{aligned}
& s_{1}=s+\mathrm{B} x \frac{\delta h}{\delta t} \pm \frac{\mathrm{S} x^{2} \mathrm{~B}}{C^{2} b^{2} h_{10}{ }^{3}} \frac{\delta s}{\delta t}+\frac{x^{2} \mathrm{~B}}{2 \log h_{0}} \frac{\delta^{2} s}{\delta t^{2}} \\
& h^{\prime}=h+\frac{s}{C^{2} b^{2} h_{01}{ }^{3}}+\frac{x}{g b h_{11}} \frac{\delta s}{\delta l}+s \frac{\delta h_{0}}{\delta l} \frac{x^{2} \mathrm{~B}}{C^{2} b^{2} h_{10}{ }^{3}} \\
& -s \frac{\delta h}{\delta t} \frac{(b+\mathrm{B})}{b^{2} g h_{0}{ }^{2}}+\frac{B x^{2}}{2 b g h_{4}} \frac{\delta^{2} h}{\delta l_{2}} \pm \frac{\mathrm{B}^{2} x^{3}}{3 \mathrm{C}^{2} b^{2} h_{l^{3}}{ }^{3}}\left(\frac{\delta h}{\delta t}\right)^{2}
\end{aligned}
$$

Avec ces formules le calcul se fait d'heure en heure et nécessite pour débuter, une base de départ. Une telle base résulte, en général, du calcul à l'aide d'une des méthodes précédentes.

Il apparaît clairement que la résolution d'un problème de marée à l'aide d'une des méthodes ci-dessus nécessite un gros travail. Comme chaque tronçon de canal donne deux équations et qu'un réseau d'ouvrages comporte souvent 20 ou 30 tronçons de canaux (fig. 1), chaque problème requiert la résolution de 40 ou 60 équations. Chaque projet peut nécessiter 30 calculs différents, de telle sorte qu'il faut afr'ronter, pour to seul calcul des marées, environ 300.000 heures de travail.

Une pratique de quinze années de calcul nous a enseigné que l'on peut maîtriser la théorie des marées, mais que l'application de cette théorie exige un déploiement d'énergie plutôt considèrable.

Ceux qui s'intéressent à l'établissement des formules ci-dessus se reporteront à la bibliographie du parag. IV. 


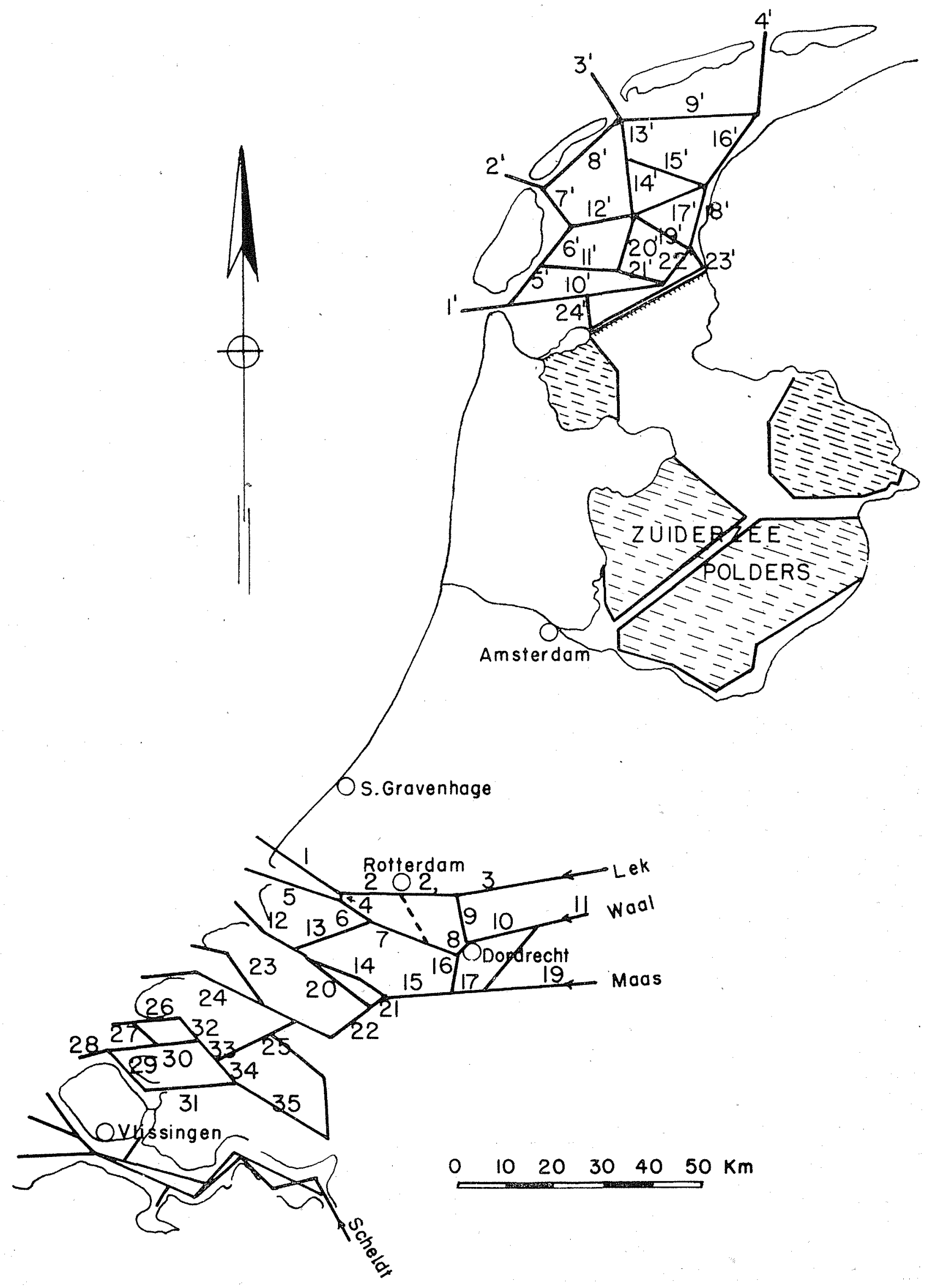

fig. 1 


\section{CHAPITRE II}

\section{L'ANALOGIE}

Le déve'oppement le plus récent est un retour à plus de simplictć au moyen des méthodes électriques. Peut-être aurait-on dî, dès l'origine, emprunter cet'e voie. Les lois de la nature soni souvent simples et belles, à condition de ne pas chercher à représenter les inoindres détails. Pour établir des projets, nous ne pouvons user de formules compliquées. Il est souhaitable que les Ingénieurs civils possedent pour les marées des formules faciles et d'applications rapides.

S'il est besoin pour le projet choisi d'une exactitude extrême, e'est un mathématicien entraîné qui fera faire ce travail.

Les principales conceptions de l'analogie, utiles à ce'ui qui désire acquérir quelques idées fondamen:ales sur les marées, sont les suivantes :

\section{1) Courants continus}

Un fleuve ordinaire sans marée, dans leque! l'écoulement est toujours de même sens, peut $\hat{e}$ re comnaré à un conducteur alimenté en courant continu.

\section{2) Courcuts alternatifs}

Un canal à marée, dans lequel les courants de flot et jusant sont soumis à une inversion périodique ressemble à un conducteur livran $i$ passage au courant électrique alternatif.

\section{3) Estuaire de fleuve à marée}

Un chenal servant d'exu'oire à un fleuve et soumis en même temps à la marée, est analogue à un conduc'eur dans lequel on établit simultanément un courant continu et un courant alternatif.

\section{4) Conductance}

Un fil gros a plus de conductance qu'un fil mince; de même un fleuve ou un canal de forte secion transversale a plus de conductance qu'une petite rivière ou qu'un petit canal. Pour des raisons qui seront éclaircies plus loin, la profondeur compte plus que la largeur. La conductance d'un profil en travers simp'e est proportionnelle à la puissance $3 / 2$ de la profondeur $h$ et à la largeur $b$, lorsqu'on considèro des profils reclangulaires. Donc, pour un large profil de rivière conductance $\infty \mathrm{b} / \mathrm{h} / \mathrm{3}$.

Les hauts-fonds comptent peu dans la conductance $b h^{3 / 2} d b$ comme le montre la fig. 2. Ce sont les parties profondes qui comptent le plus. Si l'on fait abs'raction du frottement sur les berges, un profil de $800 \mathrm{~m}$. sur I a la même conductance qu'un profil de $100 \mathrm{~m}$. sur 4, bien que l'aire du premier soit double; mise à part linfluence du frottement le long des berges, si nous doublons la profondeur d'un canal, ja conductance est multipliée par trois. Nous uliliserons cette considération par exemple pour

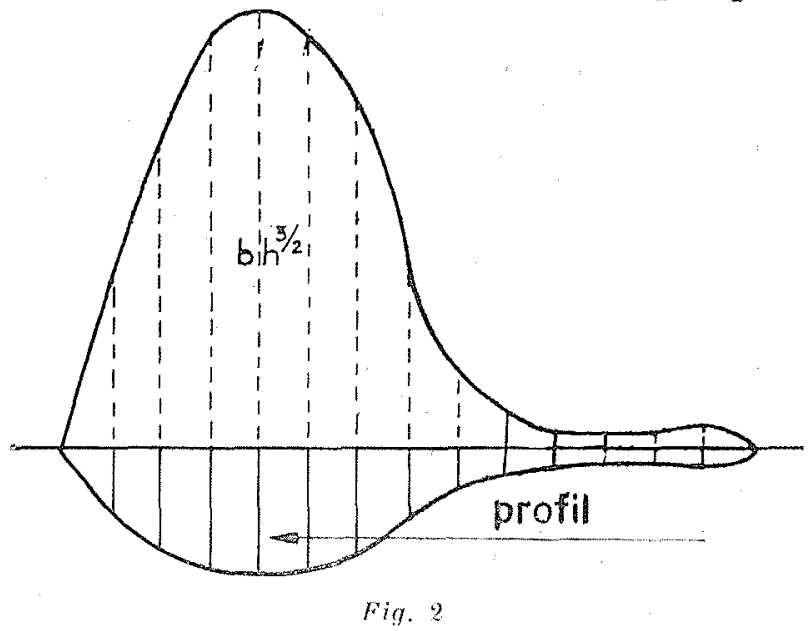

choisir la longueur de ponts en projet, en conservant la conductance normale, mais prenanl une largeur plus faible et une profondeur plus forte.

\section{5) Résistance}

Un chenal possède une « résistance de lit 》 de la même façon qu'un conducteur électrique possède une résistance propre. Ces résistances sont inversement proportionnelles à la conductance. La résistance d'un tronçon de rivière est proportionnelle à $l / b h^{3 / 4}$

Les expressions données ci-dessus pour la conductance et la résis'ance sont des expressions pratiques du domaine de l'ingénieur. Les dimensions en sont incorrectes. D'autre part elles ne concernent que des profils larges ; le cas des profils étroits est traité au parag. VIIr.

\section{6) Potentiel}

En électricité, le potentiel est l'énergie spatiale au-dessus d'un niveau de référence, le polentiel de la terre.

En hydraulique, nous pourrons de la même façon, appeler potentiel la hauteur d'un niveau d'eau au-dessus d'un p'an de référence, le niveau moyen de la mer par exemple.

L'expression « différence de potentiel 》 représente la « différence de charge » hydraulique, c'est-à-dire la diffécence de hauteur entre deux points de la ligne d'eau d'un fleuve.

Le gradient du potentiel s'assimile à la pente.

7) Puissance motrice

La puissance motrice des écoulements fluviaux est la pesanieur, exprimée par la pente ou le gradient. On fera quelque difficulté pour admettre que les écoulements de l'eau provoqués par la marée soient également attribuables à la pesanteur, car on pourrait dire que c'est l'attrac- 
tion de la lune et du soleil qui cause la marée. C'est parfaitement exact, la source réelle du mottvement périodique des océans est bien la lune (et le soleil), mais nous pouvons considérer comme des données premières, les marées de la mer aux embouchures de nos fleuves. Le mouvement vertical du niveau d'eau aux embouchures provoque des pentes et ces pentes provoquent des écoulements alternés dans un sens et dans l'autre.

Il y a une théorie qui considère les marées comme résultant d'ondes directes et réfléchies donc de deux ondes se propageant dans des directions opposées. Cette ihéorie est souvent utilisée en mathématiques; elle est également lout à fait exacte. Il n'y a qu'une différence apparenle entre cette théorie et notre conception $p^{\top} u s$ simple, selon laquelle une seule onde entre à l'embouchure et règle, par l'in ermédiaire de la pente de la surface, les courants de gravité.

\section{8) Loi d'Ohm}

En électricité la relation tres simple entre po:entiel, résistance et courant s'exprime par la loi d'Ohm :

$$
i=\frac{e}{r}
$$

ce qui signifie que pour un conducteur de longueur unité, le courant est proportionnel au gradient du potentiel et à la conductance.

En hydraulique, nous avons presque la même loi, au radical près, et cet e relation ne s'appelle pas loi d'Ohm, mais loi de Chézy:

$$
\text { courant }=\frac{\sqrt{\text { gradient de potentiel }}}{\text { résistance }}
$$

Soil $q$ le courant (en $\mathrm{m}^{3} / \mathrm{sec}$ ) $\alpha$ le gradient du potentiel, $\frac{1}{\text { C. b } h^{3 / 2}}$ la résistance, on retrouve la formule bien connue de Chézy, applicable aux canaux larges.

$$
q=\operatorname{coh}{ }^{3 / 2} \sqrt{\alpha}
$$

REMARQUE.

La formule citée est réative aux profils rectangulaires larges. Pour des profils trapézoïdaux, il faudrait la lire

$$
\eta=\operatorname{cs} \sqrt{P \alpha}
$$

dans laquelle $S$ serait la surface mouillée et $R$ le rayon hydraulique. Il n'y a pas entre ces formules de différence notable; la résistance esi

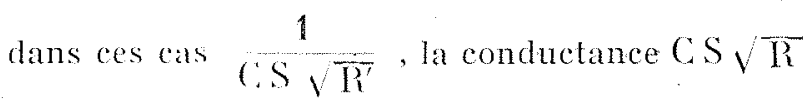

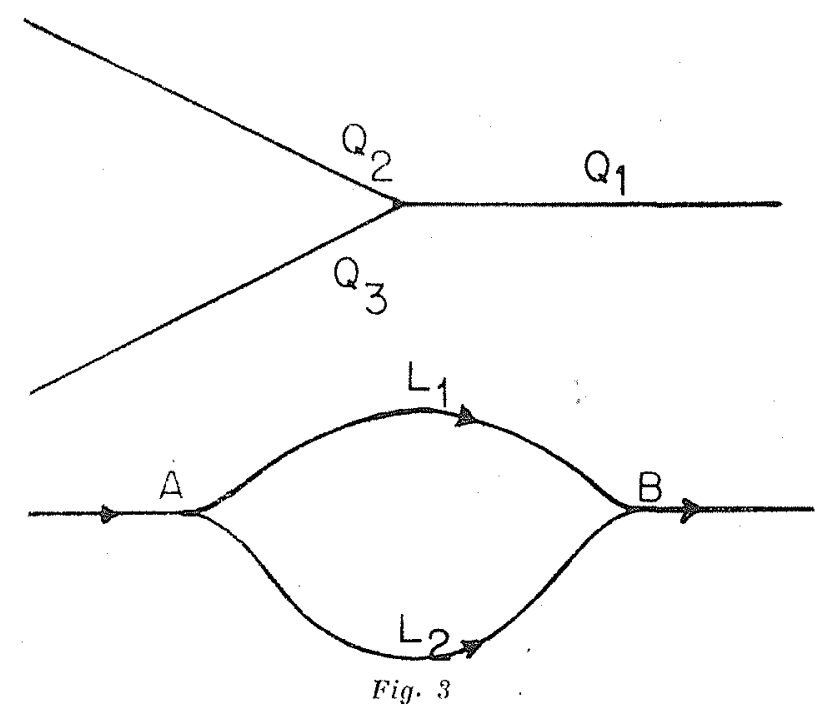

Pour des canaux plats et larges $\mathrm{S}=b \mathrm{~h}$ et $\mathrm{R}=$ $h$, alors la conduclance est $\mathrm{C} b h^{3 / 2}$.

\section{9) Loi de Kirchhoff pour des courants continus}

Au confluent de deux rivières normales (fig 3 ), les trois débis répondent à l'équation :

$q_{1}=q_{2}+q_{3}$

Si un fleuve comporte une î!e, les chutes de potentiel le long des branches 1 et 2 doivent être les mèmes. On a donc si $\alpha_{1}$ et $\alpha_{\text {: }}$ sont les pentes des deux bras, $L_{1}$ et $L_{2}$ leurs longueurs :

$\alpha_{1} \mathrm{~L}_{1}=\alpha_{2} \mathrm{~L}_{1}$

Les deux formules de Kirchholf (et la loi de Chézy ou d'Ohm) permettent de calculer facilement les débits dans les deux bras.

10) Loi de Kirchhoff pour des courants alternatifs.

Nous savons qu'en électricité les lois de Kirchhoff sont les mêmes pour les courants alernatifs que pour les comrants continus. En hydraulique, il en est de même, mais il faut remarquer que les mesures ne peuvent jamais ềre faites au point exact de jonction. Il faut considérer une certaine surface intermédiaire qu'on peut appeler Z (fig. 4). Par cycle de marée. et si A est le marnage, notre premièe loi de Kirch'off s'exprime, pour une marée venant de la droice (fig 4a), par :

$Q_{1}=Q_{2}+Q_{3}+2 \mathrm{AZ}$

Dans le cas de la fig $4 \mathrm{~b}$, on aura :

$Q_{1}=Q_{2}+Q_{3}-2 \mathrm{AZ}$

Si nous es ayons de vérifier ces formules simples, nous trouverons quelque différence avec la nature, a cause des courants d'échange entre le bras 2 et le bras 3 , et vice versa, lorsque change 


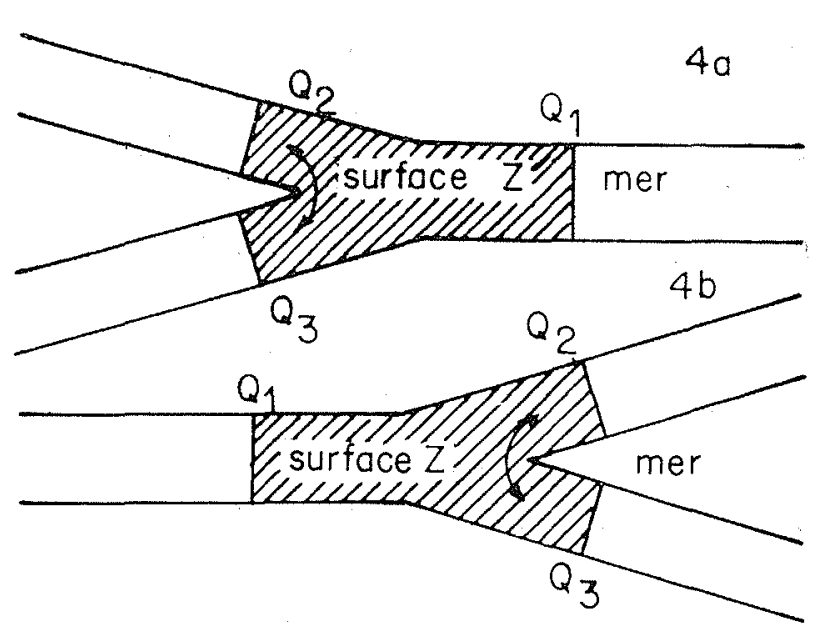

IFig. 4

la marée. Ces courants sont dus à la différence de phase de l'écoulement dans les deux bras ; on observe le mème phénomène dans le cas des courants électriques. Quand les différences de phase sont fortes, on ne peut plus négliger ces courants d'equilibrage (" corner currents").

La seconde loi de Kirchhoff pour des courants alternés (fig. 5a) revient à dire qu'il n'y a qu'une courbe de marée en chacun des points A et B. La propagation de la marée est telle que le temps de propagation le long de l'un ou l'autre côté d'une île est le mème. Nous écartons ici l'idée d'une vitesse de propagation proportionnelle à $\sqrt{g h}$; il n'en est ainsi que lorsque la théorie de deux groupes de marée opposés est à considérer.

Si nous dessinons les courbes marégraphiques de $A$ et $B$ rapportées au mème axe des temps (fig. 5b) la distance verticale de ces deux courbes à un instant donné esi $a_{1} b_{1}$, qui représente la différence de charge entre $\mathrm{A}$ et $\mathrm{B}$. Si la distance entre ces deux points est trop grande, la courbure de la ligne d'eau intervient.

A cet instant postérieur, la différence de charge $b_{2} a_{2}$ est négative; nous dirons qu'il s'agit d'une charge de jusant tandis que $a_{1} b_{1}$ était la charge de flot. Au voisinage des PM et des BM les charges deviennent temporairement nulles.

Si l'on soustrait la courbe de marée $B$ de la courbe de marée $A$, et si toutes deux sont des sinusoïdes, on obtient une nouvelle sinusoïde, décalée de 90" environ par rapport aux précédentes, et que nous pourrons appeler courbe de la différence de charge entre $A$ et $B$.

Nous appellerons aire motrice la surface de cette courbe des différences de charge entre A et $B$. Sur la fig. $5 b$, cette aire est figurée par des hachures verticales. Du fait des marées ou des courants alternés, la puissance motrice ou difference de charge n'est pas constante mais sinusoïdale (ou plus ou moins proche de la sinusoïde). Pour tout chenal il est très important de connaìtre l'aire motrice. Nous ne pouvons la connatro qu'en relevant les courbes de marée aux deux extrémités du chenal et en les rapportant exactement à la mème origine des temps. La plupart du temps, les mouvements des marémètres ne sont pas en accord avec l'heure exacte et nous ne pouvons donc pas nous fier aux aires motrices (u'on en déduit. Une différence d'une seule mintte peut encore etre trop grande.

Il résulte elairement de ce qui précède, qu'il existe une relation étroite entre la vitesse de propagation d'une marée et l'aire motrice. On comprendra que la vitesse de propagation considérée n'est pas proportionnelle à $\sqrt{g h}$ mais qu'elle est la vitesse de propagation naturelle, que donnent les marégraphes.

REMARQUE 1: Si $f$ est le volume total traversant une section d'un canal à marée pendant le flot el si $c$ est le volume analogue relatif au jusant, le volume total échangé à travers cetto section par cycle de marće est dommé (fig. 6a) par :

$$
\mathrm{Q}=\rho+e
$$

De même poux les aires motrices : aire motrice totale par cycle $=\mathrm{M}_{4}=\mathrm{M}_{2}+\mathrm{M}$

REMARQUE 2 : Si le fleuve débite, nous obtenons des figures analogues a 6 b. Au fur et a

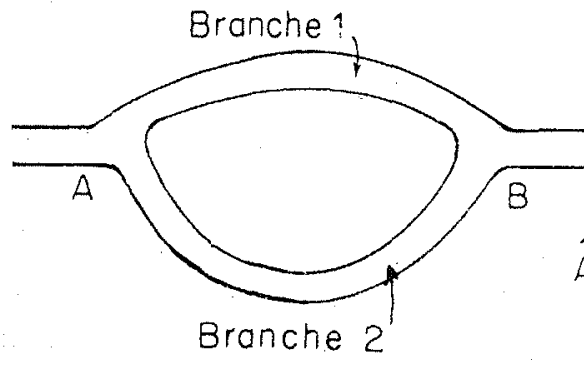

Fig. $5 a$

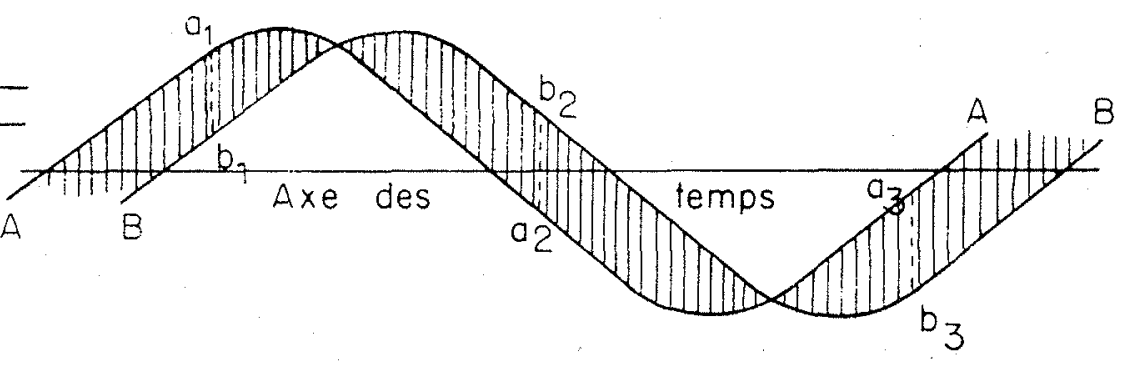

Fig. $5 b$ 
mesure que la rivière débite davantage, le volum du jusant s'accroît, le volume du flot décroît. En appelant $P$ le volume apporté par le fleuve en un cycle de marée $(44700 \mathrm{sec})$ on a les relations suivantes :

$$
\begin{aligned}
& P=e-f \\
& e=\frac{1}{2}(P+Q) \\
& f=\frac{1}{2}(Q-P)
\end{aligned}
$$

De la mème facon :

$$
\begin{aligned}
& M_{\mathrm{p}}=\mathrm{M}_{c}-\mathrm{M}_{i} \\
& \mathrm{M}_{c^{\prime}}=\frac{1}{2}\left(\mathrm{M}_{\mathrm{p}}+\mathrm{M}_{0}\right) \\
& \mathrm{M}_{b}=\frac{1}{2}\left(\mathrm{M}_{0}-\mathrm{M}_{\mathrm{p}}\right)
\end{aligned}
$$

REMARQUE 3: A une grande distance de la mer, il n'y a plus de flot notable. Les courbes des courants et des forces motrices deviennent semblables à celles dessinées fig. $6 \mathrm{c}$ on $6 \mathrm{~d}$.

\section{1) Self - Induction.}

Dans la thérie des courants alternatifs, le facteur $\cos \varphi$ est de grande importance, il est souvent voisin de 0,9 . Pour une période, on a en électricité :

$$
i_{n}=\frac{e_{m}}{r_{m}} \cos \varphi
$$

formule dans laquelle $i_{m}$, $e_{m}$ et $r_{m}$ sont des moyennes étendues au cycle entier. $\varphi$ est appelé angle de déphasage et $\cos \varphi$ est le facteur de self-induction.

Quel est, en hydraulique, le phénomène analogue à la self-induction ? C'est l'inertie des masses d'eau, par suite de laquelle ces masses, ne s'arrêtent pas de couler au moment où la pente s'annule. L'écoulement maximum ne se produit pas en mème temps que la pente maximum mais avec un décalage $\varphi$. Dans la science des marées, nous aussi nous avons l'angle de déphasage et le facteur de self-induction. La plupart du temps, également, ce demier est voisin de 0,9 pour nos canaux. Dans les mers ouvertes, il est bien moindre.

La relation entre les courbes de marée (dites courbes des cales), la courbe des pentes (dite courbe motrice) et la courbe de courant dite courbe horizontale dans un certain profil est celle que montre la fig. 7. Nous appellerons respectivement ces 3 courbes : courbe a, courbe $\alpha$ el courbes.
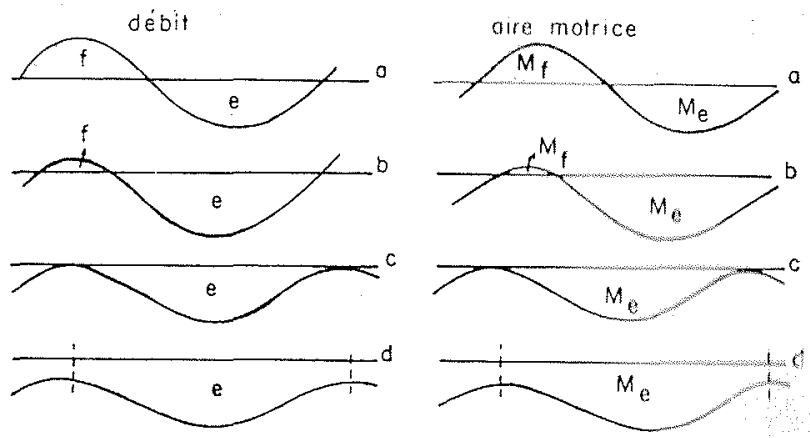

Fig. 6

I.a courbe des pentes a coupe l'axe des pen. tes nulles aux PM et aux BM et passe par des maxima ou minima aux points $A_{1}$, $A_{2}$ et $A_{3}$, on lesquels la courbe «a $»$ (courbe de la marée verticale) coupe la ligne zéro.

La courbe $s$ des courants est déphasée en arrière de la courbe $\alpha$.

La fig. 7, de la plus grosse importance, se trouve dans tout traité d'électricité élémentaire. On devrait aussi la trouver dans tous les lives élémentaires sur les marées. La relation très simple liant les courbes $a, \alpha$ et $s$ doit etre une évidence pour tout ingénieur qui s'occupe d'estuaires ou de fleuves à marce.

12) Loi dOhm pour les courants a mare

La formule de l'électricité

$$
i_{m}=\frac{e_{\mathrm{m}}}{r_{\mathrm{m}}} \cos \varphi
$$

relative à une période complète trouve son pendant dans la formule suivante, relative aux courants au cours d'un cycle de marée:

$$
\begin{gathered}
Q=c_{n} b_{m} h_{m}{ }^{32} \times \sqrt{\alpha^{m} \tau \cos \varphi} \\
\text { (pour un cycle) }
\end{gathered}
$$

Ce n'est pas seulement le fait d'introduire dans la formule de Chézy un facteur de self-induction qui rend cette nouvelle formule valable pour un eycle complet de 44.700 secondes, mais $\delta$ plus $\alpha_{m}$ n'est pas une valeur particulière de la pente

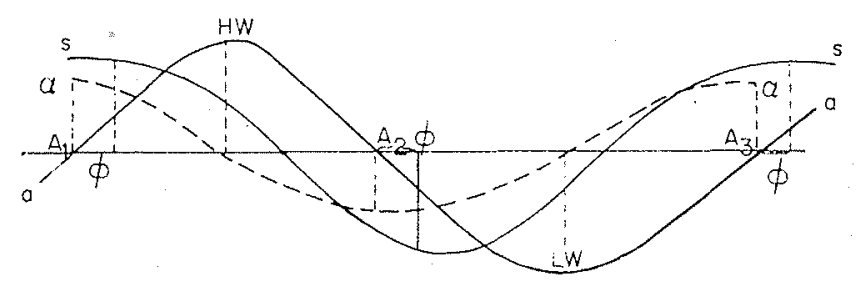

Fiq. 7 

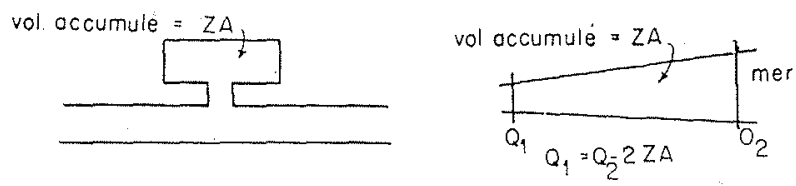

Fig. 8

mais une aire motrice, ou plutot un gradient moteur. Si $M$ est l'aire motrice entre 2 staions de mesure et si $l$ est leur distance, le gradient moteur est $\alpha_{m} \ldots \frac{\mathrm{M}}{l} \quad$ D'autre part $b_{m}$ est la largeur moyenne de la section mouillée et $h{ }_{m}$ la profondeur moyenne durant le cycle.

Quiconque prendra la peine de vérifier cette nouvelle formule en mesurant le volume total écoulé et le gradient moteur (ce dernier aussi exactement que possible), pourra constater que notre conception est exacte. On pourra trouver $\varphi$ en mesurant l'intervalle de lemps qui sépare la PM ou la BM du plus proche instant d'étale des courants conséculifs.

On remarquera que la formule ci-dessus ne fait intervenir qu'un profil.

\section{3) Condensateur et capacité}

Un port ouvert de surface $Z$ peut accumuler une quantité d'eau $A Z, A$ étant l'ampitituds de la marée. Le volume d'eau échangé par cycle de marée à travers son débouché est $2 \mathrm{AZ}$. Un port ouvert est un condensateur de capacité $A Z$.

Un canal à marée possède une capacié propre,

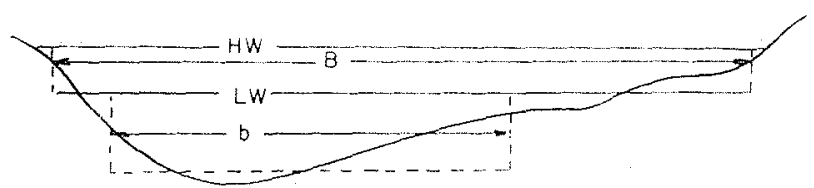

Fig. 9

de la même façon qu'un conducteur électrique a comme disent les électriciens, une capacité r'épartie.

Pour un troncon de canal a marée de longueur $l$, on a la relation suivante (fig. 8):

$$
\begin{aligned}
& \mathrm{Q}_{1}=\mathrm{Q}_{2} \pm 2 \mathrm{ABl} \cos \varphi \\
& \text { (formule de remplissage) }
\end{aligned}
$$

Dans cette formule $B$ est la largeur moyenne du canal plein. Elle est toujours supériecire à la largeur de l'écoulement $b$, comme on peut aisément le voir fig. 9.
Le facteur cos $\varphi$ introduit le fait que le phénomène de remplissage d'un canal à marée ne doit pas être considéré entre les niveaux de PM et de BM mais entre les niveaux correspondanis aux instants d'étale des courants. Cependant, nos mesures ne sont pas, d'ordinaire, suffisamment exactes pour permettre une vérification de cette formule de nature à mettre en évidence I'influence de $\cos \varphi \cdot \operatorname{Cos} \varphi$ est souvent voisin de 0,9 ; pour des culs-de-sac à marée, $\cos \varphi=1$; pour des mers profondes, $\cos \varphi$ est à peu près nul. Dans ce cas, la capacité ne compte pas du tout.

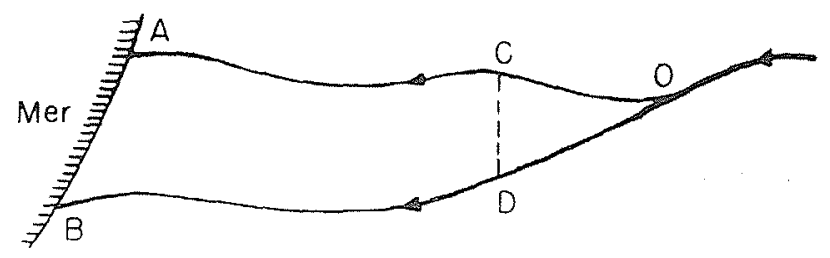

Fig. 10

La formule simple du remplissage découle, par intégration pour un cycle entier, de l'équation de continuité meniionnée.

Les électriciens ne peuvent pas négliger les capacités réparties des grands conducteurs, teis que les càb'es télégraphiques ou téléphoniques. C'est pourquoi ils utilisent l'équation des télégraphistes qui est tout à fait analogue à nos écruations de LoRENTz (chapitre I).

\section{4) Pont de Weatstone}

\section{Fleuves normaux ou collants continus :}

On peut considérer un fleuve muni de deux affluents, comme nous l'avons dessiné fig. 10. Si l'on faisait un canal entre deux points $C$ et D au même potentiel ou au même niveau, il n'y aurait aucun écoulement dans ce canal AB. $A B$ est un «pont de Weatstone».

Courants alternatifs dans des canaux à marées

Considérons le cas de 2 canaux voisins AA, et $B B_{1}$, (fig. 11) comporlant 2 sections $C$ et

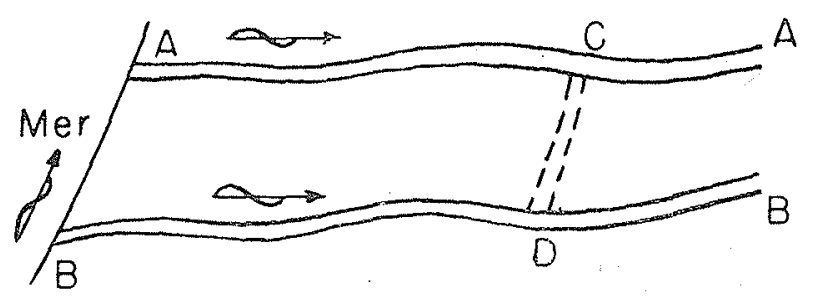

Fig. 11 
D où la marée est de même amplitude et de même phase. Alors il n'y aura jamais aucune force motrice entre les points $C$ et $D$ et, par conséquent, pas d'autre courant que celui dû au remplissage de $C D$. On rencontre souvent des canaux qui s'approchent de la conception du pont de Weatstone. Ces canaux sont p!us ou moins parallèles à la côte et des améliorations du projet, en un endroit quelconque du réseau, réagissent très sévèrement sur l'écoulement dans ces canaux. Elles provoquent de nombreux changements et des envasements. Si l'on améliore le canal CA, l'écoulement dans le canal $C D$ peut, selon la nouvelle phase de la marée en $C$, cesser presque complètement ou prendre une importance bien plus grande qu'auparavant.

A vrai dire, on ne rencontre jamais « un pont de Weatstone » parfait, aux deux extrémités duquel les marées verticales seraient exactement égales et de même phase, mais de nombreux canaux sont proches de la situation critique correspondant à un envasement ou à une érosion. Ce sont des «ponts de Weatstone » ou quelque chose d'approchant.

\section{5) Facteur d'activité}

Par suite de la « capacité répartie » des canaux à marée, les profils en travers doivent devenil plus grands vers la mer. Les berges des canaux à marée s'évasent ou doivent s'évaser en allant vers la mer.

Ia loi d'Ohm n'étant valable que pour des profils en travers isolés, nous introduirons, de manière à pouvoir traiter des tronçons de canaux, le « facteur d'activité " $\mathrm{F}$ :

$$
F=\frac{Q}{b h^{3 / 2}}
$$

II est souhaitable que ce facteur $F$ soit constant pour tous les profils d'un troncon de canal.

De (6) et de la loi d'Ohm (4) on déduit immédiatement :

$\mathrm{F}=\mathrm{C} \sqrt{\alpha_{m} \mathrm{~T} \cos \varphi}=\mathrm{C} \sqrt{\frac{\mathrm{M}}{1} \mathrm{~T} \cos \varphi}$

De cette formule résulte que si $\mathrm{F}$ est constant dans une partie d'un canal, il en est de mème du gradient moteur $\mathrm{M} / \mathrm{e}$ ou de la vitesse moyenne (C et $\varphi$ varient peu).

L'expression « facteur d'activité », choisie pour $\mathrm{F}$ cherche à exprimer le fail qu'une forte valeur de $F$ est un signe d'acion érosive tandis qu'une faible valeur de $F$ indique probablement qu'une sćdimentation est en cours.

res trois courbes representant, le long du
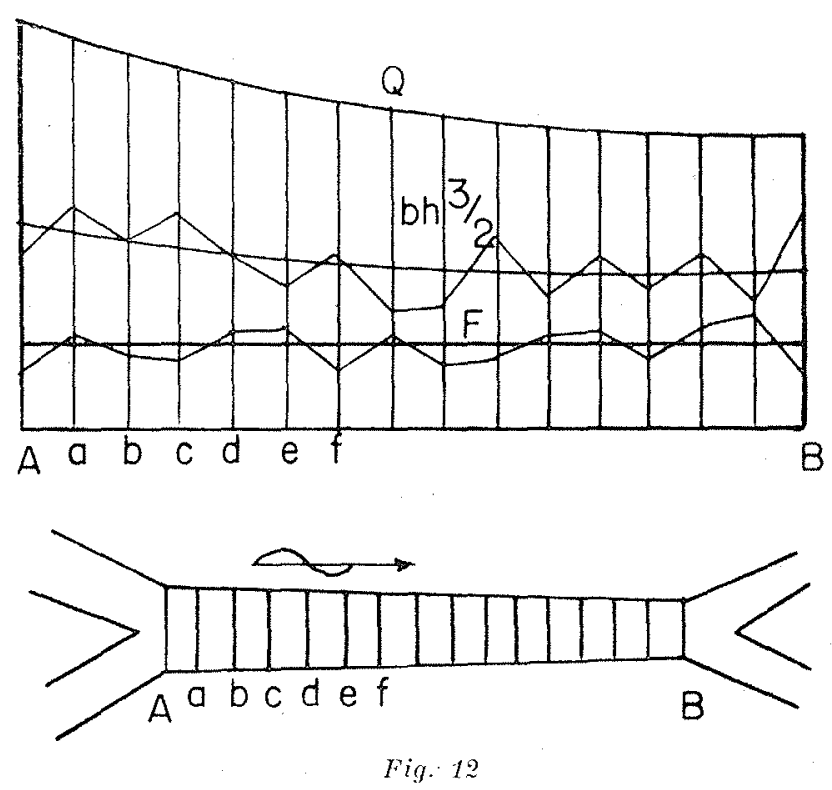

chenal, le volume total échangé $Q$, la conduclance $b h^{3 / 2}$ et le facteur d'activité F ressenblent souvent plus ou moins à celles de la fig. 12. Les deux premières sont croissantes d'amont en aval tandis que le facteur d'activité reste ou doil rester plus ou moins constant.

Dans un réseau de canaux soumis ou non à la marée, on ne peut changer la valeur du $F$ d'aucun canal sans changer les valeurs de $F$ de tous les autres canaux. Il est important d'avoir un réseau avec de bons facteurs d'activité, car l'envasement ou l'érosion en dépendent.

\section{6) Formule de l'évasement}

Le Dr Herbert ChatLey a donné, dans le $N^{\circ} 71$ de « Selected Engineering papers of the Institution of Civil Engineers », la formule suivante :

$$
\lambda=2 \frac{\mathrm{B}^{2} \mathrm{~A}}{\mathrm{Q}}
$$

formule dans laquelle $\lambda$ est l'évasement par' kilomètre et $Q$ le volume total d'eau échangé par cyc'e au travers d'un profil. Cette formule est basée sur une profondeur moyenne $h$ constante et peut être obienue, à partir de la formule de capacité, de la manière suivante :

On $\mathrm{a}: \mathrm{Q}_{1}=\mathrm{Q}_{2}+2 \mathrm{AB} l \cos \varphi$

Ceci peut s'écrire (fig. 8) :

$$
\begin{gathered}
C_{1} B_{1} h_{1}: \sqrt{\alpha_{1} \tau \cos \varphi_{1}} \quad C_{2} B_{2} h_{2}^{3 / 2} \sqrt{\alpha_{2} \tau \cos \varphi_{2}} \\
=2 A B l \cos \varphi
\end{gathered}
$$

Ici $B$ est assimilé au $b$ de la fig. 9.

Si l'on suppose $\mathrm{C}_{1}=\mathrm{C}_{2}=\mathrm{C} ; \alpha_{1}=\alpha_{2}=\alpha$; $\cos \varphi_{1}=\cos \varphi_{2}-\cos \varphi=1 ; h_{1}=h_{2}=h ;$ 


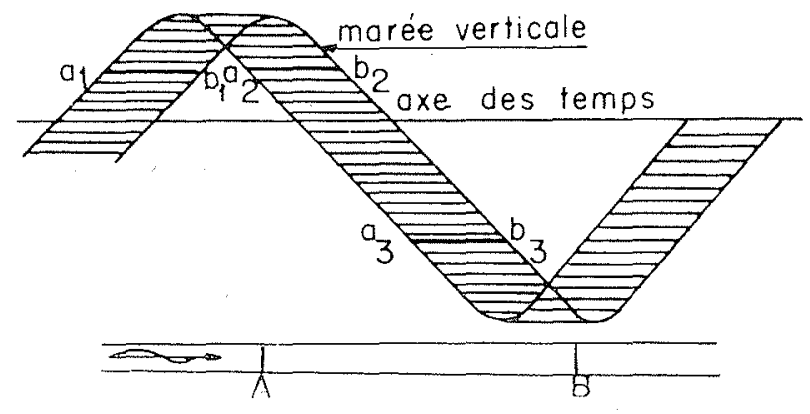

Fig. 13

on en déduit :

$$
C h=\sqrt{\alpha \tau}\left(B_{1}-B_{3}\right)=2 A B l
$$

ou, avec : $\lambda=\frac{B_{1}-B_{2}}{l}$

$$
\lambda=\frac{2 \mathrm{BA}}{C h^{3 ! 2} \sqrt{\alpha \mathrm{T}}}=\frac{2 \mathrm{~B}^{-} \mathrm{A}}{Q}
$$

Cette formule de Chatley est souvent trop simple parce que $b$ diffère de $B$ et $h_{1}$ de $h_{2}$. En général, on cherche à introduire la constante $\mathrm{F}=\frac{\mathrm{Q}}{b h^{2}} \cdot$ Alors la formule de capacité devient :

$$
\frac{b_{1} h_{1} b^{3}-b_{2} h_{2}}{l}=\frac{2 \mathrm{AB} \cos \varphi}{\mathrm{F}}
$$

Cela indique que l'accroissement de conductance par km. doit être proportionnel à la largeur moyenne B en surface et au double de l'amplitude de la marée, et inversement proportionnel au coefficient d'activité.

L'évasement peut donc ètre pris arbitrairement ou selon les circonstances, tandis que les profondeurs peuvent être calculées d'après ce choix.

\section{7) Propagation de l'onde de marée}

Comme on l'a dit précédemment, la vitesse de propagation de l'onde de marée est en rapports étroits avec l'aire motrice et, par consé-

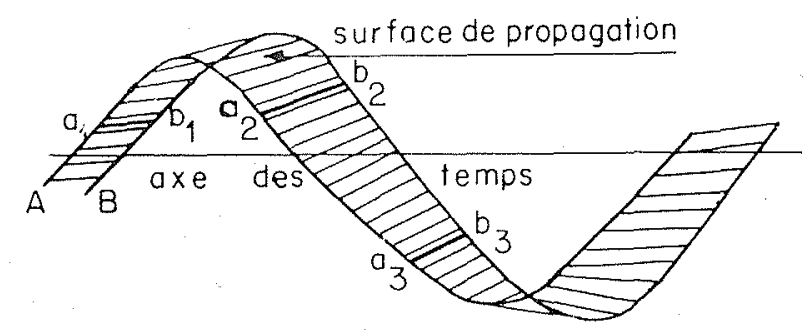

Fig. 14 quent, avec l'écoulement. Cela demande quelques compléments d'explications.

S'il y a propagation uniforme (fig. 13) la courbe des marées en $\mathrm{A}$ est iclentique à la courbe des marées en B, mais de phase différente.

Dans ce cas, on a pour les segments horizontaux :

$$
a_{1} b_{1}=a_{2} b_{2}=a_{3} b_{3}, \text { etc... }
$$

Le temps 'T' de propagation est le même pour' tous les points de la marée verticale. Si l est la distance entre les deux stations $A$ et $B$, on a pour la célérité :

$$
C=\frac{l}{\mathrm{~T}}
$$

S'il $y$ a propagation non uniforme (fig. 14) la vitesse de propagation n'est pas constanle aux différents moments de la marée. Cependanl, nous pouvons considérer sa moyenne plus ou moins donnée par :

$$
\text { C.mogen }=\frac{M}{H .700} \mathrm{~m} / \mathrm{sec} \text {. }
$$

Si la propagation de l'onde de marée est rapide, les pentes sont faibles et les courants rela-

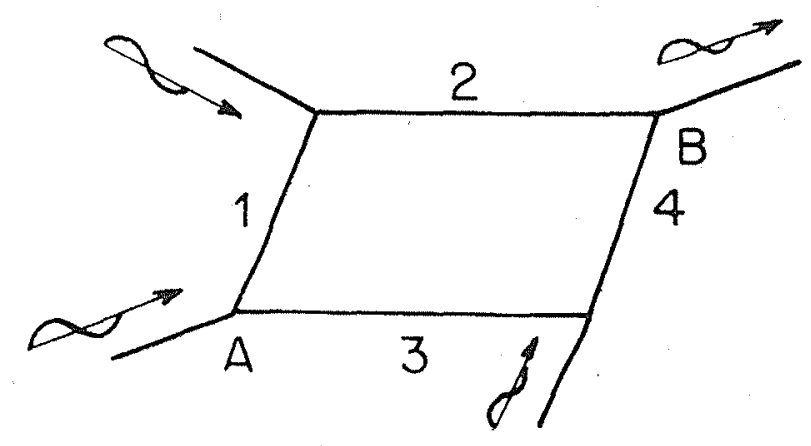

Fig. 15

livement faibles. Si la propagation de la maréc est difficile et lente, les pentes sont fortes et, par conséquent, les courants rapides. On peut done désigner à première vue sur n'importe quelle carte de lignes cotidales, les emplacements des courants violents; ils se situent la où les lignes cotidales sont très proches les unes des autres.

18) Propagation commandée par les lois d'Ohm et de Kirchhoff

La formule si souvent utilisée à tort :

$$
\mathrm{V}=\mathrm{v} \sqrt{g h}
$$

qui indique une proportionnalité entre la vitesse de propagation, la racine de la profondeur et 
l'accélération de la pesanteur, n'est pas du tout propre à nos calculs d'application. On ne devrait jamais employer cette formule, à moins qu'on ne désire appliquer la difficile théorie des chassés-croises d'ondes, qu'on utilise si l'on fait des mathématiques plus élevées. La formule en question n'est valable que pour les ondes dites libres.

II est facile d'expliquer que, dans quelques cas, la vitesse de propagation peut ètre tout à fait indépendante de la profondeur du canal, par exemple dans le cas d'un «pur pont de Weatstone $»$ : dans ce cas, la vitesse de propagittion est infinie tandis que la profondeur ne joue plus.

Dans tout réseau de canaux à marée, il ne peut y avoir qu'une marée verticale en chaque noud. Pour une maille telle que celle de la fig. 15 , on a done, en appelant $\mathrm{T}$ les temps de propagation :

$$
\mathrm{T}_{1}+\mathrm{T}_{2}=\mathrm{T}_{3}+\mathrm{T}_{1}
$$

Nous pouvons également écrire :

$$
\mathrm{H}_{1}+\mathrm{M}_{2}=\mathrm{M}_{3}+\mathrm{M}_{1}
$$

car les aires motrices sont comprises entre les courbes de marée en $A$ et $B$.

Si nous mettons à part l'inertie, 3 causes régissent les écoulements arec ou sans marée :

1) la force motrice ou pente ou vitesse de propagation.

2) la résistance.

3) le courant.

Aucune d'entre elles n'est indépendante des autres; la nature les introduit ensemble dans une certaine relation, la loi d'Ohm. Cette loi e! la loi de Kirchhoff régissent la réunion des ondes de marée en chaque noud de nos réseaux de canaux à marée.

Dans le cas d'une énorme maille telle que celle qui englobe l'ensemble Angleterre-Ecosse, il n'est pas possible à l'onde qui fait le tour de l'Ecosse d'arriver au rendez-vous en même temps que l'onde qui a traversé la Manche. Cependant, la nature y a pourvu, en fournissant un point de rencontre parfait près de la côte du Kent et en faisant arriver l'onde venant du Nord avec exactement un cycle de déphasage. L'onde arrivant du Nord devant la côte du Kent n'y rencontre done pas sa sœur, mais sa nièce. De mème, autour d'un point amphidromique, tei que l'un des deux points amphidromiques de la mer du Nord, l'onde de marée est telle qu'il faut exactement un cycle de marée à l'onde venant du sud pour faire le tour de ce point afin de rencontrer sa nièce qui vient d'y arriver. On peut comparer ces points amphidromiques à de petites îles au centre d'une baie dont la marée pourrait faire le tour en un cycle de marée complet.

\section{9) Tendances à gauche (ou a droite) des embouchures}

Dans un estuaire le chenal le plus profond ef le plus large se trouve du còté d'où vient la marée. Par exemple, le long des côtes de Hollande et d'Allemagne baignées par la mer du Nord, les exutoires de tous les principaux chenaux se trouvent vers le Sud-Ouest ou l'Ouest. Notre théorie permel d'expliquer facilement cette préférence. Soit un fleuve à marée se terminant par deux défluents; la marée se propage dans le sens AB (fig. 16). Le chenal AC doit

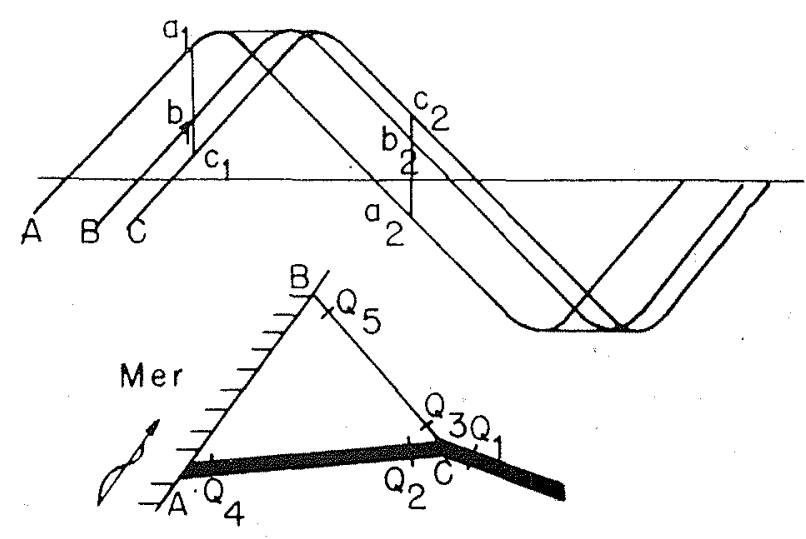

Fig. 16

avoir, dans ce cas, un profil en travers plus important que le chenal $\mathrm{BC}$. On peut le voir en traçant, par rapport au même axe des temps, les courbes des marées verticales aux stations $A, B$ et C. A marée montante, la différence de charge $a_{1} c_{1}>b_{1} c_{1}$ et de même à marée descendante $a_{2} c_{2}>b_{2} c_{2}$.

Nous pouvons aussi écrire:

$$
\mathrm{N}_{u}>\mathrm{M}_{w}
$$

Il y a dans le canal AC une force motrice plus forte que dans le canal $\mathrm{BC}$, donc aussi des cou* rants de flot ou de jusant plus intenses, conduisant à des profils plus grands.

De faibles différences d'amplitude entre $A$ et $B$ ne modifient pas la «tendance à gauche », comme on le voit en traçant des figures telles que 16. Sur la cote allemande de la Mer du Nord, l'accroissement d'amplitude a lieu vers l'Ouest, 

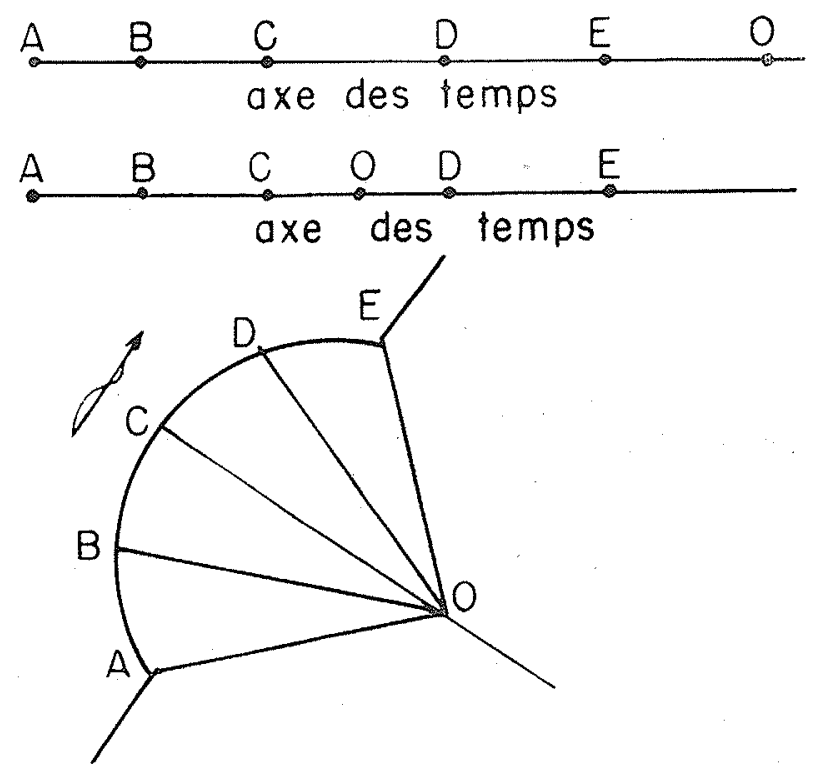

Fig. 17

mais il n'influe que très faiblement sur la tendance à gauche de l'écoulement.

A vrai dire, il faudrait aussi considérer la force de Coriolis qui, dans l'hémisphère Nord, tend à dévier l'écoulement sur sa droite, (et aussi bien les courants de jusant que de flo:), mais généralement cette force a peu d'influence.

Les aires motrices, ou plutôt les gradients moteurs (c'est-à-dire les aires motrices par kilomèire), sont les principaux facteurs à considérer.

Plus forte est la surface motrice le long de la côte entre $A$ et $B$, plus forte est la tendance à gauche. Si un même fleuve possède 3 embouchures (fig. 17), on peut représenier les phases moins selon les conductances et la racine des gradients moteurs en ces embouchures.

$\mathrm{Si}$ la marée verticale varie peu aux embouchures (fig. 17), on peut représenter les phases par de simples points portés sur l'axe des temps. à raison d'un seul point par station. En supposant que le décalage de phase de la marée verticale en tête du delta (point 0 ) soit le plus fort, les aires motrices aux 5 embouchures sont proportionnelles aux temps $\mathrm{AO}, \mathrm{BO}, \mathrm{CO}, \mathrm{DO}$ et EO. En supposant que la phase de la marée verticale en $O$ se situe entre $C$ et $D$, les aires motri ces sont également proportionnelles à $\mathrm{AO}, \mathrm{BO}$ etc..., mais on voit que lorsque l'écoulement a lieu vers la mer, dans les canaux DO et EO, il a lieu en sens contraire dans les canaux $A O$, $\mathrm{BO}$ et $\mathrm{SO}$.

\section{0) Exemples pratiques}

Comme nous l'avons dit tout à fait au début, deux formules sont à employer :

l'équation dynamique et l'équation de continuité.

Les formes les plus simples auxquelles on peut les ramener sont la loi d'Ohm (4) et l'équation de remp'issage (5). Toutes deux se réfèrent à un cycle complet et ne sont pas intrinsèquement enchevêtrées comme el'es devraient l'être. C'est pourquoi l'on ne peut aborder, avec leur concolirs, que des équations simples. Dans les cas où la consiruction des ouvages ne modifie pas l'amplitude de la marée verticale, on peut utiliser ces formules simples; par contre elles ne doivent pas ètre utilisées dans les cas plus importants où l'amplitude doit considérablement changer. Il est très instructif, pour les débutants, d'utiliser en premier la méthode la plus simple. Généra'ement les marées verticales sont bien développées dans les réseaux et de petites modifications du profil d'un ou de plusieurs canaux n'affectent pas sévèrement l'amplitude. Les changements principaux concernent toujours les courants, les frottements et la propagation. Les marnages changent rarement beaucoup el s'il le faut on peut, au départ des calculs, esimer ces changements.

On devrait commencer par mesurer les coefficients de Chézy $C$ dans tous les canaux, en utilisant de très bons chronomètres pour relever les courbes de marée verticale avec leurs phases exactes. Dans le systeme métrique on trouve 52 pour valeur moyenne de $C$ dans les parties aval des fleuves de Hollande.

Nous pouvons également mesurer $\cos \varphi$ dans tous les canaux. Comme valeur moyenne de $\varphi$ on trouve $35^{\circ}$, soit 0,9 pour $\sqrt{\cos } \varphi$. Ce dernier facteur varie entre 0,87 et 0,94 pour différents canaux.

En raison des variations assez faibles de $\mathrm{C}$ et de $\cos \varphi$ pour l'ensemble d'un réseau d'ouvrages, on pent les réunir au facteur $\sqrt{44700}$ qui intervien lausi à chaque fois dans le calcul. On obtient alors le très agréable facteur

$$
52 \sqrt{ } 44.700 \times 0,9=10.000
$$

L'on peut alors utiliser les formules extrêmement simples :

$\mathrm{F}=10.000 \sqrt{\frac{\mathrm{M}}{\mathrm{l}}}$ équation dynamique

et

$Q_{1}=Q_{3} \pm 2 \mathrm{AB} /$ équation de continuité 
Cependant il n'est pas sûr que d'autres estuaires se prêtent à l'emploi du même facteur simple 10.000. Seules des mesures de l'écoulement et des jaugeages exacts peuvent permettre de vérifier ce chiffre.

Il n'est pas prudent de considérer de trop longs tronçons de fleuves. Pour des f'euves de $400 \mathrm{~m}$. de large environ, la longueur des troncons ne doit pas dépasser $15 \mathrm{~km}$; pour ceux de $1.000 \mathrm{de}$ large environ, la longueur peut, sans beaucoup d'inconvénient, dépasser cette valeur. La stylisation doit êlre faite avec soin. C'est avec de l'expérience seulement que l'on peut alteindro l'exactitude maxima.

Ensuite nous devons évidemment atiliser les lois simples de Kirchhoff. Il faul alors considérer 4 formules différentes : la loi d'Ohm, déjà citée, la formule de remplissage et les deux lois Kirchhoff. Leur utilisation domne la connaissance des principes auxquels obéit la marée dans tout estuaire ou réseau d'ouvrages avec ou sans marée (par exemple un réseau dirrigation) mais ces formules ne donnent pas l'ultime détail et ne doivent pas avoir le dernier' mot quand il faut établir des plans importants.

On trouvera, ci-après, quelques exemples.

\section{1) Exemple d'un canal unique'}

Le calcul le plus simple est relatif à un canal unique $A B$ qui coupe une péninsule entourée d'une mer à marée (fig. 18).

Supposons que les courants dans le nouveau canal ne modifient pas les marées verticales dans la mer près de $A$ el de $B$. Alors l'aire motrice $\mathrm{M}_{\mathrm{a}}$ peut être évaluée exactement; elle ne changera pas du fait de l'exécution du nouveau canal. Le volume total échangé par eycle résulte immédiatement, dans ce cas simple, de la formule:

$$
Q=10.000 b h^{3 \cdot 2} \sqrt{\frac{\mathrm{M}_{a b}}{l_{a b}}}
$$

Si l'on admet que le débit instantané $q$ dans $A B$ suit une loi sinusoïdale, on trouve facilement :

$$
\varphi \max =\frac{\pi \times Q}{2 \times 44.700}
$$

Si l'on suppose que les marées en $A$ el $B$ changent après exécution du canal, on doit englober dans le calcul les deux troncons supplémentaires AC et BD (fig. 18) alors c'est $\mathrm{M}_{(, 1)}$ qui constitue notre condition aux limites sans changement. Les facteurs recherchés

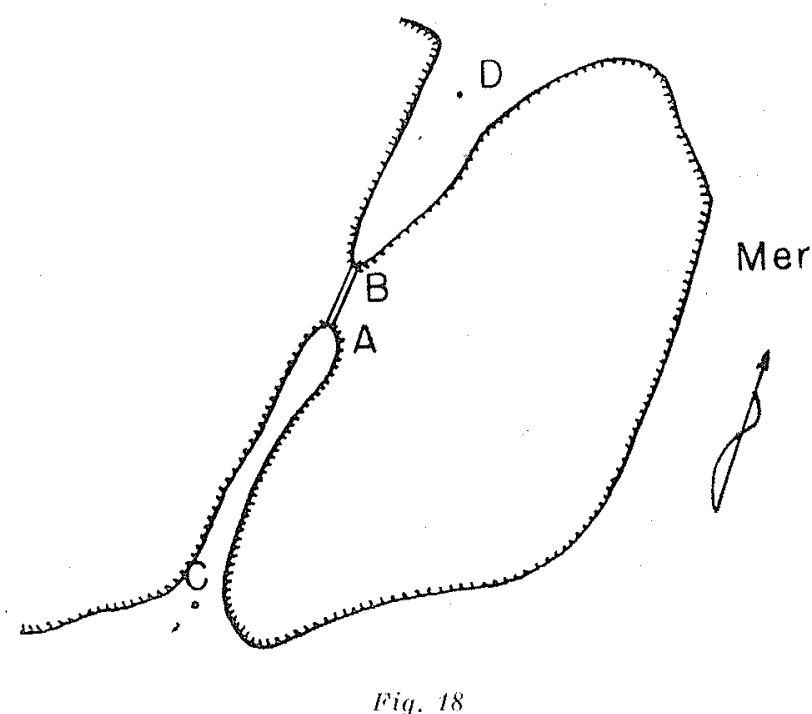

sont : $M_{a c}, M_{a b}, M_{b d}, Q$. Ces quatre facteurs requièrent la résolution des équations suivantes :

$$
\begin{aligned}
& \mathrm{M}_{a c}+\mathrm{M}_{a b}+\mathrm{M}_{b d}=\mathrm{M}_{d c} \\
& \mathrm{~F}_{a b}=\frac{\mathrm{Q}}{b_{1} \cdot h_{\mathrm{t}}}=10.000 \sqrt{\frac{\mathrm{M}_{a b}}{l_{a b}}} \\
& \mathrm{~F}_{a t}=10.000 \sqrt{\frac{\mathrm{M}_{a c}}{l_{a c}}} \\
& \mathrm{~F}_{b d}=10.000 \sqrt{\frac{\mathrm{M}_{b d}}{l_{a b}}}
\end{aligned}
$$

22) Calcul de la « lendance à gauche *

Pour un fleuve à marée possédant deux embouchures (fig. 16) les condivions aux limites sont l'aire motrice $\mathbf{M}_{\text {it }}$, le long de la côte et la marée horizontale du fleuve à l'amont, $Q \ldots$ On en déduit les formules qui suivent :

$$
\begin{aligned}
& Q_{2}+Q_{3}=Q_{c} \\
& M_{a c}+M_{b c}=M_{a b} \\
& F_{a c}=10.000 \sqrt{\frac{M_{a c}}{l_{a c}}} \\
& F_{b c}=10.000 \sqrt{\frac{M_{b c}}{l_{b c}}}
\end{aligned}
$$

Si les profils en travers sont connus, on peut calculer les courants ; si l'on se donne une vitesse maximum de $x \mathrm{~m} / \mathrm{sec}$. en chaque embouchure, on peut calculer les dimensions des profi's. Il faut utiliser la formule de capacité pour calculer tous les profils le long de $\mathrm{AC}$ et $\mathrm{BC}$ 
23) Calcul d'un nonveau canal en rinnissant deux autres

S'il s'agit d'un nouveat canal réunissant deux fleuves à marée existants et parallèles (fig. 19) on trouve les formules suivantes :

On conniait $Q_{1}, Q_{2}$, et $M_{\text {itd }}$ et l'on suppose que l'amplitude de la marée ne changera pas du fait du creusement du nouveau chenal. On a :

$$
\begin{aligned}
& \mathrm{Q}_{1}=\mathrm{Q}_{3}+\mathrm{Q} \\
& \mathrm{Q}_{2}=\mathrm{Q}_{;}+\mathrm{Q} \\
& \mathrm{M}_{a t}=\mathrm{M}_{a b}+\mathrm{M}_{b c}+\mathrm{M}_{a d} \\
& \mathrm{~F}_{a b}=10.000 \sqrt{\frac{\mathrm{M}_{a b}}{l_{a b}}} \\
& \mathrm{~F}_{b c}=10.000 \sqrt{\frac{\mathrm{M}_{b c}}{l_{b c}}} \\
& \mathrm{~F}_{c d}=10.000 \sqrt{\frac{\mathrm{M}_{c d}}{l_{c d l}}}
\end{aligned}
$$

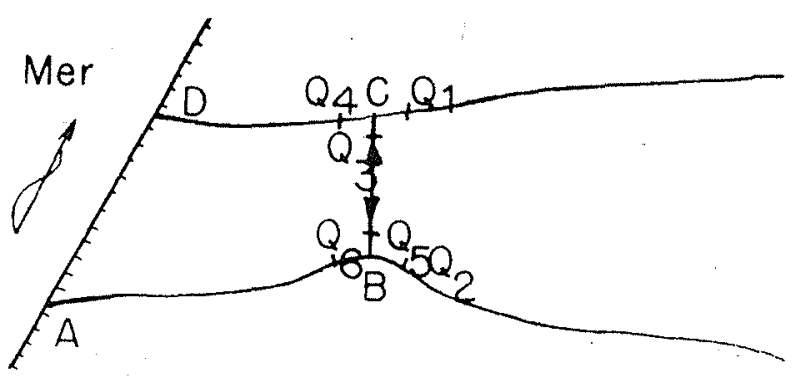

Fig. 19

Il faut utiliser les formules de remplissage pour obtenir les écoulements dans tous les profils le long de AB, BC et DC.

On traitera tout autre exemple de cette façon pourvu que l'on suppose inchangée, du fait des travaux, l'amplitude de la marée verticale.

L'écoulement du fleuve à l'amont n'a qu'une incidence faible sur la valeur de $\cos \varphi$, comme l'ont montré les mesures sur les bouches du Rhin. $\operatorname{Cos} \varphi$ varie graduellement de 0,9 à 1 au fur et à mesure que l'on se rapproche de la limite d'action de la marée à l'amont, mais en pratique cette faible différence se remarque difficilement. Le volume total écoulé, la résistance et la propagation peuvent se calculer sans prê- ler une attention particulière à l'ćcoulement du fleuve à l'amont.

\section{CHAPITRE III}

\section{METHODE D'IMITATION ELECTRIQUE}

Si l'on recherche une exactitude plus grande, il faut utiliser l'une des méthodes plus exactes, mais plus fastidieuses. Ces méthodes abordent aussi bien le cas de la marée verticale que celui de la marée horizontale, mais c'est la méthode dite exacte qui donne le plus grand nombre de détails et qui est la plus précise, car elle tient compte de la relation quadratique entre vitesse et frottement.

Afin d'éviter l'énorme travail qu'elle implique, on peut représenter le réseau de canaux à marée par des conducteurs en cuivre, des résistances électriques, des condensateurs, etc..., en alimentant simultanément ce système au moyen d'un courant alternatif et d'un courant continu. If va sans dire que la méthode linéaire de Lorentz peut ètre représentée très facilement par une méthode électrique, car cette méthode est tout à fait analogue aux formules des télégraphistes.

On trouvera, fig. 20, la photographie d'une telle représentation d'un fleuve à marée, le Leck, pour lequel la marée vient de la gauche et meurt vers la droite à l'endroit où se produit l'appor! des eaux du fleuve. L'exactitude de cette analogie électrique est, comme on peut s'y attendre, la même que celle des réponses obtenues à partir des formules, car l'analogie est précisément faite selon ces formules. La longueur totale du fleuve représentée est de $90 \mathrm{kms}$

Isinconvénient de la méthode linéaire ne réside pas seulement dans ses résultats insuffisants lorsque l'on recherche une forte précision, mais aussi dans le fait qu'elle nécessite de nombreux calculs préliminaires pour déterminer la constante de Lorentz, K proportionnelle à la vitesse $V$, inconnue de prime abord. La représentation électrique de marées quadratiques ne nécessite pas ces calculs préliminaires. La difficulté de reproduction électrique d'une marée quadratique réside évidemment dans lo fait qu'il faut recourir à un dispositif électrique pour obtenir la relation quadratique entre courant et frottement. Des courants électriques purs donnent seurement la relation linéaire. Cependant, la radiotechnique moderne fournit des moyens de transformer électriquement cette relation. L'exactitude obtenue à l'aide de 


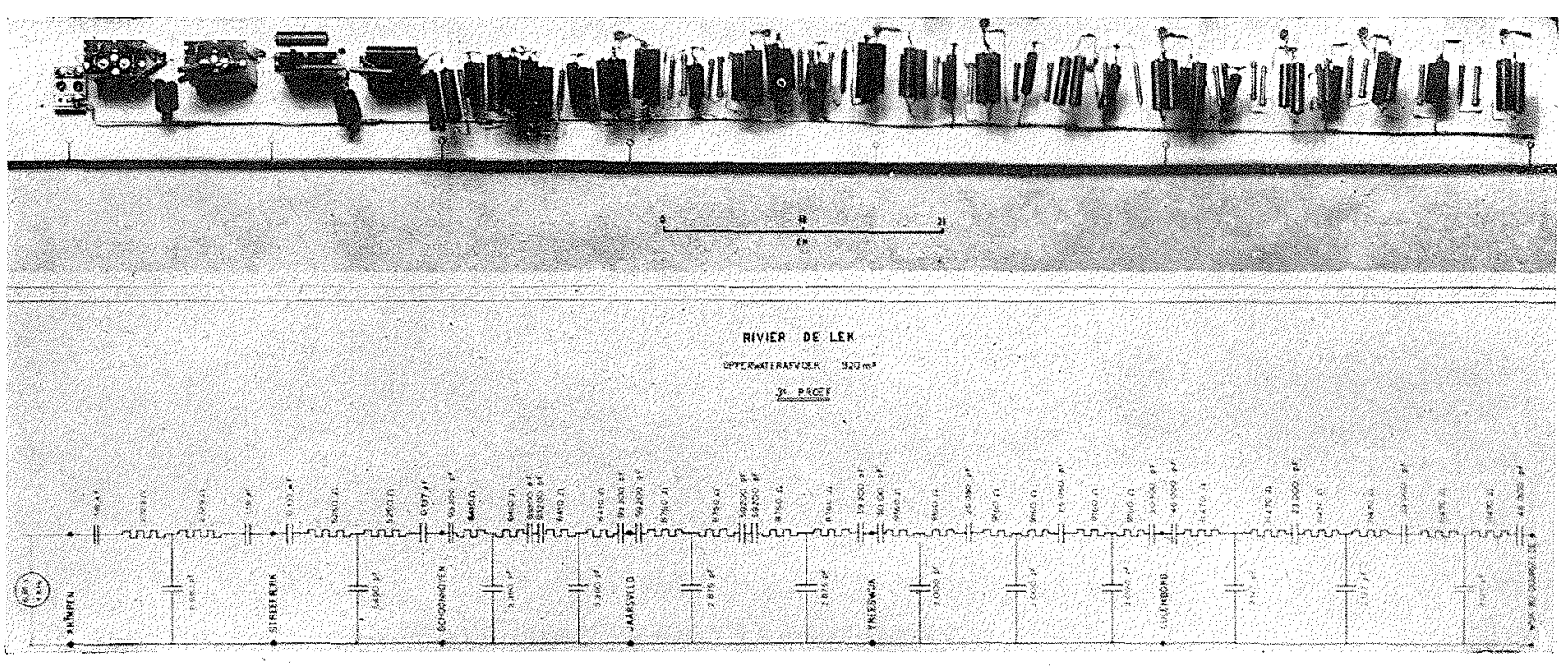

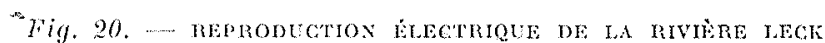

lanalogie électrique est d'environ $1 / 2 \%$ par rapport aux formules.

A l'aide d'un tube à rayons cathodicues, on peut observer à l'oil les nouvelles marées verticales, ou les photographier. On peut mème uliliser un lube à doubles rayons avec lequel on peut observer deux marées, et la relation exacte entre les formes et leurs phases, avec tous les détails et particularités des futures courbes de marées (fig. 21 et 22 ).

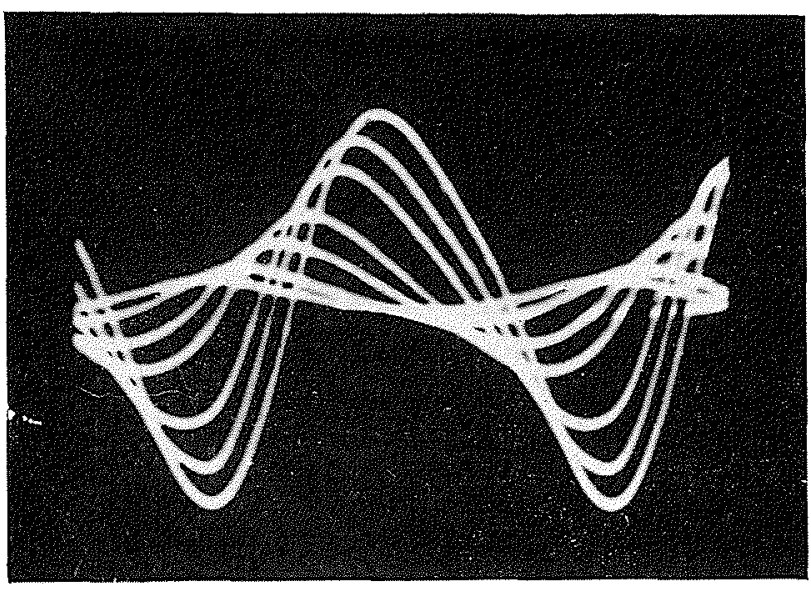

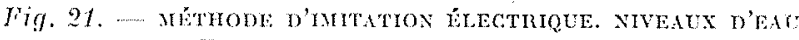
DU TECK BN FONCTION DU TFMPS.
Bien que les moyens électroniques visent à éliminer beaucoup de calculs ardus, nous mettrons en garde le lecteur contre le fait de négliger la thérie de marées. On pourrait commettre des fautes involontaires si la connaissance actuelle des fleuves et la recherche de cette connaissance devaient décroitre par suite de l'emploi des méthodes trop faciles pour traiter les problèmes d'estuaires à marée.

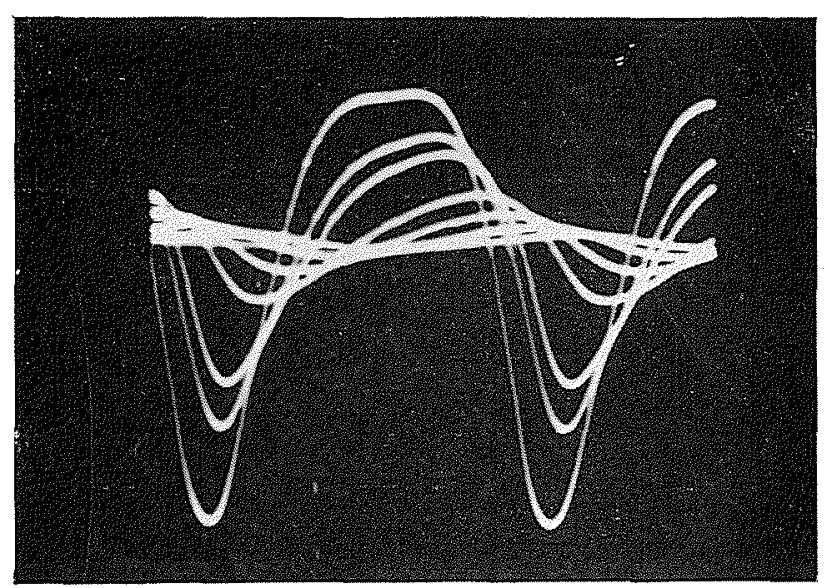

Fig. 22. - MÉthode D'mitation flectriour.

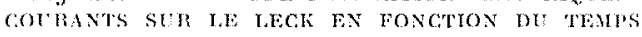




\section{CHAPITRE IV}

\section{BIBLIOGRAPHIE}

H. A. LorENT'z : Verslag van de Staatscommissie 1918 ter afsluiting van Zuiderzee, Den Haag - Algemeene Landsdrukkerij 1926 336 pages, 61 fig.

J. J. Dronkers : Een getijberekening vool
Benedenrivieran, De Ingénieur 1935 - * pages, 3 fig.

J. VAN VEen : Getijstroomberekening mel behulp van wetten analoog met die van Ohm en Kirchhoff - 1937 - De Ingenieur $\mathrm{n}^{\circ} 19$ 9 pages - 16 fig.

J. M: MAZURE : De berekening van getijfen en stormvloeden op benedenrivieren. Gerritsen Den Haag - 1937 - 222 pages ,- 12 fig.

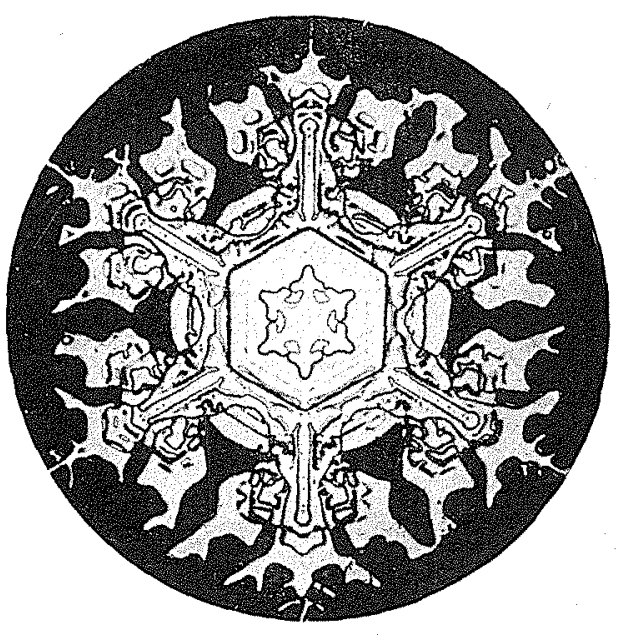

